\title{
Research Paper: The Serum Level of Midkine in Patients With Multiple Sclerosis and Neuromyelitis Optica
}

\author{
Milad Moayednia $^{1} \odot$, Leila Dehghani ${ }^{2 *}$, Amir Safi ${ }^{1} \odot$, Vahid Shaygannejad ${ }^{1} \odot$, Karim Sohrabi $^{1}$ O, Majid Rezvani ${ }^{3} \odot$, Mohammad Reza \\ Akrami $^{3}$, Mojgan Soghrati ${ }^{1}$, Mohammad Mahdi Aboūtalebi ${ }^{1} \odot$, Mahdi Barzegar ${ }^{1} \odot$ \\ 1. Isfahan Neurosciences Research Center, Alzahra Research Institute, Isfahan University of Medical Sciences, Isfahan, Iran. \\ 2. Department of Tissue Engineering and Regenerative Medicine, School of Advanced Technologies in Medicine, Shahid Beheshti University of Medical \\ Sciences, Tehran, Iran. \\ 3. Department of Neurosurgery, Kermanshah University of Medical Sciences, Kermanshah, Iran.
}

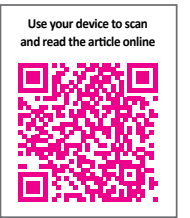

Chtation: Moayednia, M., Dehghani, L., Safi, A., Shaygannejad, V., Sohrabi, K., \& Rezvani, M., et al. (2021). The Serum Level of Midkine in Patients With Multiple Sclerosis and Neuromyelitis Optica. Basic and Clinical Neuroscience, 12(2), 199-204. http:// dx.doi.org/10.32598/bcn.12.2.1009.2

http://dx.doi.org/10.32598/bcn.12.2.1009.2

Article info:

Received: 15 Aug 2019

First Revision: 10 Oct 2019

Accepted: 25 Feb 2020

Available Online: 01 Mar 2021

Keywords:

Midkine, Multiple sclerosis, Neuromyelitis optica

\section{A B S T RA C T}

Introduction: Midkine (MK), a heparin-binding growth factor, is involved in neurological diseases by mediating the inflammatory responses through enhancing the leukocyte migration. The present study assesses the serum concentration of this growth factor among newly developed Multiple Sclerosis (MS) and Neuromyelitis Optica (NMO) patients.

Methods: The present research, as a cross-sectional study, was performed at Isfahan University of Medical Sciences, Isfahan City, Iran. All samples were selected from patients who visited Kashani and Alzahra hospitals for two years (2014 to 2016). The MK level was assessed in 80 new MS cases, $80 \mathrm{NMO}$ patients, and 80 healthy subjects. After collecting blood sera samples, MK serum level was measured using the ELISA. The obtained data were analyzed in SPSS.

Results: The Mean \pm SD MK level was $1038.58 \pm 44.73 \mathrm{pg} / \mathrm{mL}$ in the MS group, which was significantly higher than the Mean \pm SD MK level in the NMO $(872.62 \pm 55.42 \mathrm{pg} / \mathrm{mL})$ and control groups $(605.02 \pm 9.42 \mathrm{pg} / \mathrm{mL})$.

Conclusion: Overall, these results demonstrated that MK plays a prominent role in inflammatory reactions and neuroautoimmune diseases, especially in MS. So, the MK level may be used for earlier diagnosis and also prevention of disease progression by using a special inhibitor.

*Corresponding Author:

Leila Dehghani, PhD.

Address: Department of Tissue Engineering and Regenerative Medicine, School of Advanced Technologies in Medicine, Shahid Beheshti University of Medical Sciences, Tehran, Iran.

Tel: +98 (31) 36201250

E-mail:leiladehghani@azh.mui.ac.ir 


\section{Highlights}

- The mean Midkine (MK) level in Multiple Sclerosis (MS) patients is higher than Neuromyelitis Optica (NMO) patients and the healthy control group.

- The level of MK is higher in the NMO group compared with the healthy one.

- The healthy subjects have the lowest mean of MK level compared with the other groups.

- Sex and age have no significant association with the MK level.

\section{Plain Language Summary}

Multiple Sclerosis (MS) and Neuromyelitis Optica (NMO) are two distinct diseases with similar clinical symptoms, so it is necessary to distinguish these disorders from each other. Although there are many studies to find biomarkers for this goal, only a few of them have been validated for routine clinical practice. Because of the inflammatory nature of MS and NMO, our research group claims to evaluate the utility of Midkine (MK) as a heparin-binding growth factor that is implicated in inflammation. Our results demonstrated that MS patients have higher MK levels compared to NMO patients and the healthy participants. Also, sex and age have no significant association with the MK level. Therefore, MK may be a reliable biological marker in differentiating between MS and NMO.

\section{Introduction}

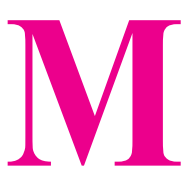

ultiple Sclerosis (MS) is a chronic autoimmune neurological disease of the Central Nervous System (CNS) (Ghasemi, Razavi, \& Nikzad, 2017) that leads to inflammatory demyelination of axons

(Gourraud, Harbo, Hauser, \& Baranzini, 2012). Neuromyelitis Ooptica (NMO, Devic's syndrome) is another immune-mediated chronic inflammatory disorder of the CNS that predominantly targets the optic nerve and spinal cord (Pandit et al., 2015). Distinguishing between the two diseases has become increasingly important because the treatments differ, and Interferon-beta (IFN- $\beta$ ) can make NMO worse (Papadopoulos, Bennett, \& Verkman, 2014). NMO can usually be differentiated from MS by the presence of serum immunoglobulin IgG autoantibody (NMO-IgG) against the astrocytic water channel Aquaporin-4 (AQP4) (Lennon et al., 2004). Although it constitutes a sensitive and highly specific biomarker, it is challenging to diagnose negative NMO patients for this marker from MS patients (Matthews et al., 2013). However, despite many studies which claim to have discovered markers for potential use, only a few clinically useful biomarkers have been successfully validated for routine clinical practice (Comabella \& Montalban, 2014).

Midkine (MK), also known as the heparin-binding growth factor, is a cytokine implicated in various pathological conditions ranging from neurodegenerative dis- eases to inflammation and cancer (Muramatsu, 2010). MK expression is enhanced during induction and progression of experimental autoimmune encephalitis, the animal model of MS (Weckbach, Muramatsu, \& Walzog, 2011). Because of the need for inflammatory markers available in MS and NMO diseases, the current study aimed at investigating the serum levels of $\mathrm{MK}$ in patients with MS and NMO and also healthy controls.

\section{Methods}

\subsection{Subjects and samples}

In this cross-sectional study, the subjects were selected among newly diagnosed MS cases based on McDonald criteria (2010) and the patients with newly diagnosed NMO according to the Wingerchuk criteria (2006). They were referred to the MS clinics of Alzahra and Kashani hospitals in Isfahan City, Iran. They were the patients who had no concomitant inflammatory diseases and no history of steroid or immunosuppressive medication. Individuals with infectious disease, blood abnormalities, and autoimmune disease were excluded. The control group was chosen from non-first-degree relatives of the patients and hospital staff who did not have any neurological problems. Each group consisted of 80 patients from both sexes, who were between 16 and 55 years old.

Blood samples were taken from all subjects in the case and control groups. After centrifugation, serum speci- 


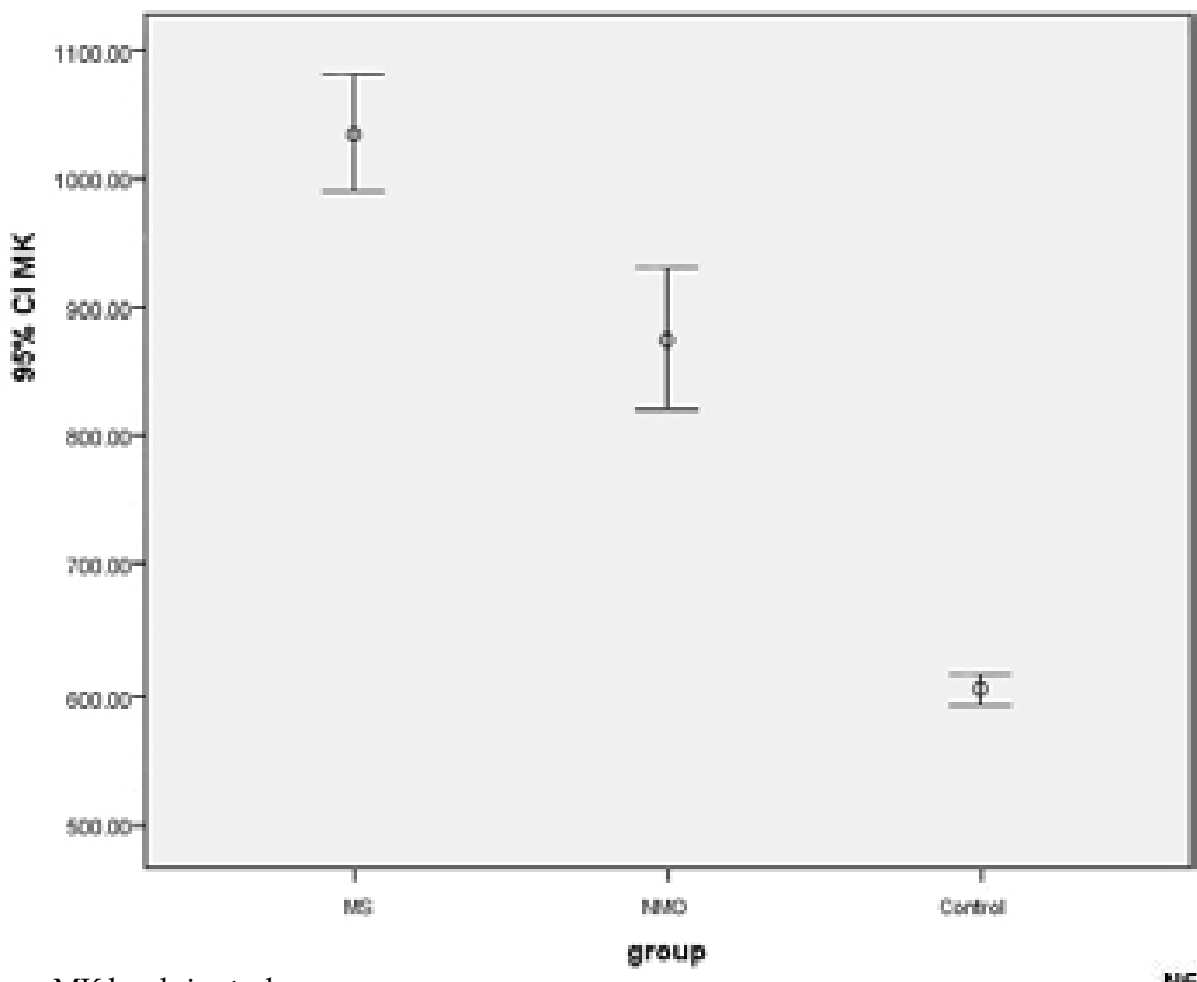

Figure 1. The mean MK levels in study groups

NEUR JSCIENCE

mens were isolated and stored frozen at $-20^{\circ} \mathrm{C}$ until further analyses. The serum MK level was measured by Enzyme-Linked Immunosorbent Assay (ELISA) using an MK kit (Glory Science, USA). The concentration of MK in the samples was determined by comparing the Optical Density (OD) of the samples at $450 \mathrm{~nm}$ to the standard curve.

\subsection{Statistical analyses}

All analyses were performed using IBM SPSS Version 25 . The significance level was 0.05 , and the data were expressed as Mean $\pm \mathrm{SD}$. One-way Analysis of Variance (ANOVA) followed by Tukey's multiple comparison test was used to compare the mean MK level among the three groups.

\section{Results}

The characteristics of the study cases are summarized in Table 1. All groups were matched in terms of age and gender; therefore, there was no significant difference between the three groups $(\mathrm{P}>0.05)$

The main results are summarized in Table 2. There was a statistically significant difference between the mean MK level among the three groups so that the MS group had the highest mean compared to the NMO and control groups $(\mathrm{P}<0.0001)$. The data are shown in Figure 1.

By pairwise comparisons of the three groups, Tukey's test revealed that the Mean \pm SD MK level was $163.523 \pm 66.59$ in the MS group, which was significantly higher than that in the NMO group $(\mathrm{P}<0.0001)$. Also, it was observed that the Mean \pm SD MK level was significantly higher in the MS group than the control group

Table 1. Demographic data of individual study subjects

\begin{tabular}{cccccc}
\hline \multicolumn{2}{c}{ Parameters } & MS $(\mathbf{n}=\mathbf{8 0})$ & NMO $(\mathbf{n = 8 0})$ & Control $(\mathbf{n = 8 0})$ & P \\
\hline \multicolumn{2}{c}{ Age, $y$, Mean \pm SD } & $33.60 \pm 9.91$ & $34.77 \pm 10.26$ & $34.83 \pm 10.07$ & 0.687 \\
Sex, No. (\%) & Male & $26(32.5)$ & $28(35.0)$ & $30(37.5)$ & 0.803 \\
& Female & $54(67.5)$ & $52(65.0)$ & $50(62.5)$ & \\
\hline
\end{tabular}




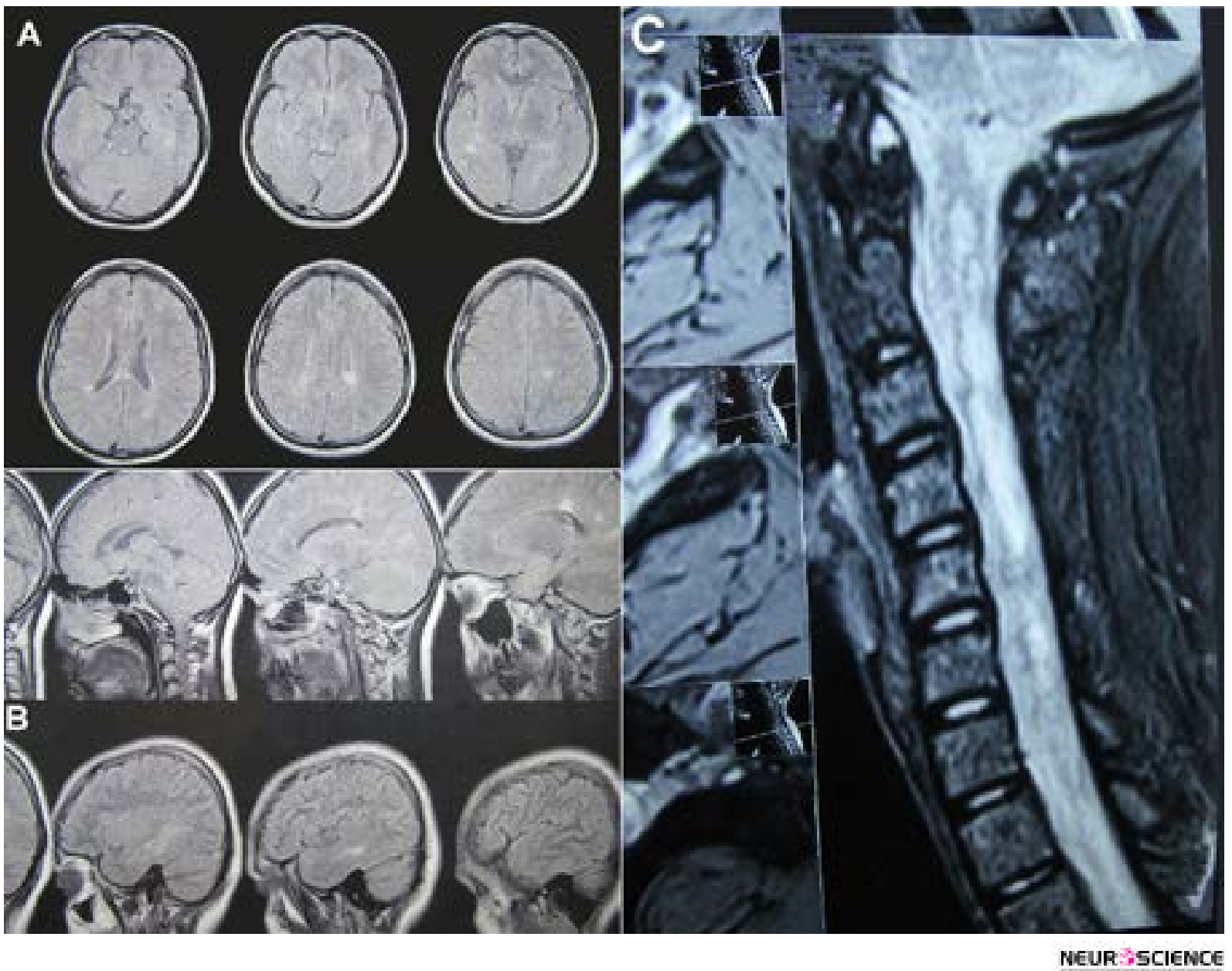

Figure 2. Brain and spinal cord MRI

A and B: The MRI brain of 26 years old female with MS; C: The MRI spinal cord of 14 years old female with NMO.

(433.565 \pm 66.59$)(\mathrm{P}<0.039)$ and NMO than the control group (270.042 \pm 66.59$)(\mathrm{P}<0.0001)$. No significant difference was reported among the three studied groups in the mean MK level according to age and sex $(\mathrm{P}>0.05)$, as presented in Table 2. Brain and spinal cord MRI are shown in Figure 2.

\section{Discussion}

Based on our results, although MS and NMO are both autoimmune disorders and have the same clinical manifestations, the inflammatory responses are more prominent in patients with MS. Moreover, age and sex do not have any significant association with MK levels. Increased levels of MK in autoimmune and inflammatory diseases have been shown in previous studies.

Our result was consistent with the study of Salama et al., which reported that MK levels in patients with Alzheimer disease, as another neurological disease, are higher than that in healthy persons (Salama et al., 2005).
Plasma MK was elevated in patients with systemic lupus erythematosus, which was correlated with Interleukin (IL)-17 (Wu, Yuan, Pan, \& Ye, 2017). In Rheumatoid Arthritis (RA) patients, high MK levels were implicated in the pathogenesis of RA and were associated with IL-6 and IL-8 (Maruyama, Muramatsu, Ishiguro, \& Muramatsu, 2004; Shindo et al., 2017). In acute and chronic kidney injuries like ischemia/reperfusion injury and diabetic nephropathy, MK expression was upregulated and promotes migration of neutrophils and macrophages; hence MK deficiency may show less renal damage (Kosugi et al., 2007; Sato et al., 2001). In inflammatory bowel diseases, including ulcerative colitis and Crohn's disease, serum MK level was positively associated with disease activity index (Krzystek-Korpacka, Neubauer, \& Matusiewicz, 2009; Krzystek-Korpacka, Neubauer, \& Matusiewicz 2010).

Our previous study indicated that MS patients who received IFN- $\beta$ treatment had a lower level of MK and IL23 than healthy controls, which has a contradiction with our recent research performed on newly diagnosed MS 
Table 2. The Mean \pm SD MK level with respect to sex, age in study groups

\begin{tabular}{|c|c|c|c|c|}
\hline Groups & & & Mean $\pm S D$ & $\mathbf{P}$ \\
\hline \multirow{8}{*}{ MS } & \multirow{3}{*}{ Sex } & Male & $1081.04 \pm 405.56$ & \multirow{3}{*}{0.316} \\
\hline & & & & \\
\hline & & Female & $1018.14 \pm 399.59$ & \\
\hline & \multirow{5}{*}{ Age (y) } & $<25$ & $1066.99 \pm 237.54$ & \multirow{4}{*}{0.901} \\
\hline & & & & \\
\hline & & $25-35$ & $1008.74 \pm 458.86$ & \\
\hline & & $>35$ & $1038.45 \pm 429.33$ & \\
\hline & & MK & $1038.58 \pm 44.73$ & 0.0001 \\
\hline \multirow{8}{*}{ NMO } & \multirow{3}{*}{ Sex } & Male & $768.96 \pm 304.37$ & \multirow{3}{*}{0.431} \\
\hline & & & & \\
\hline & & Female & $928.44 \pm 567.95$ & \\
\hline & \multirow{4}{*}{ Age (y) } & $<25$ & $863.12 \pm 333.92$ & \multirow{4}{*}{0.474} \\
\hline & & & & \\
\hline & & $25-35$ & $970.28 \pm 596.11$ & \\
\hline & & $>35$ & $808.33 \pm 481.45$ & \\
\hline & \multicolumn{2}{|c|}{ MK } & $872.62 \pm 55.42$ & 0.0001 \\
\hline \multirow{8}{*}{ Control } & \multirow{3}{*}{ Sex } & Male & $597.13 \pm 77.88$ & \multirow{3}{*}{0.551} \\
\hline & & & & \\
\hline & & Female & $609.76 \pm 88.32$ & \\
\hline & \multirow{4}{*}{ Age (y) } & $<25$ & $614.87 \pm 86.39$ & \multirow{4}{*}{0.392} \\
\hline & & & & \\
\hline & & $25-35$ & $585.95 \pm 77.13$ & \\
\hline & & $>35$ & $613.93 \pm 87.99$ & \\
\hline & \multicolumn{2}{|c|}{ MK } & $605.02 \pm 9.42$ & 0.0001 \\
\hline
\end{tabular}

patients. It reveals that the serum level of this marker increases in neurodegenerative diseases in their early onset. So that assessing MK level might help evaluate the prognosis of the disease stage (Shaygannejad et al., 2014).

It can be concluded that the MK level in newly diagnosed MS and NMO patients was more than that in the healthy individuals. The higher MK level might lead to inflammatory cytokine induction and the development of neuronal cells and, therefore, disease progression. Although our study was a cross-sectional survey, it can be a beginning for cohort assessment and further investigation.

\section{Ethical Considerations}

Compliance with ethical guidelines

Written informed consent was obtained from all participants, and the research protocol was approved by the Medical Ethics Committee of Isfahan University of Medical Sciences.

Funding

This study was supported by the Isfahan Neurosciences Research Center, Alzahra Research Institute, Isfahan University of Medical Sciences (No. 292224).

Authors' contributions

All authors contributed to preparing this article. 


\section{Conflict of interest}

The authors declared no conflict of interest.

\section{References}

Ghasemi, N., Razavi, S., \& Nikzad, E. (2017). Multiple sclerosis: pathogenesis, symptoms, diagnoses and cell-based therapy. Cell Journal (Yakhteh), 19(1), 1-10. [DOI:10.22074/cellj.2016.4867] [PMCID] [PMID]

Gourraud, P. A., Harbo, H. F., Hauser, S. L., \& Baranzini, S. E. (2012). The genetics of multiple sclerosis: An up-to-date review. Immunological Reviews, 248(1), 87-103. [DOI:10.1111/j.1600065X.2012.01134.x] [PMID] [PMCID]

Pandit, L., Asgari, N., Apiwattanakul, M., Palace, J., Paul, F., \& et al., \& GJCF International Clinical Consortium \& Biorepository for Neuromyelitis Optica (2015). Demographic and clinical features of neuromyelitis optica: A review. Multiple Sclerosis Journal, 21(7), 845-53. [DOI:10.1177/1352458515572406] [PMID] [PMCID]

Papadopoulos, M. C., Bennett, J. L., \& Verkman, A. S. (2014). Treatment of neuromyelitis optica: State-of-the-art and emerging therapies. Nature Reviews Neurology, 10(9), 493. [DOI:10.1038/ nrneurol.2014.141] [PMID] [PMCID]

Lennon, V. A., Wingerchuk, D. M., Kryzer, T. J., Pittock, S. J., Lucchinetti, C. F., \& Fujihara, K., et al. (2004). A serum autoantibody marker of neuromyelitis optica: Distinction from multiple sclerosis. The Lancet, 364(9451), 2106-12. [DOI:10.1016/S01406736(04)17551-X]

Matthews, L., Marasco, R., Jenkinson, M., Küker, W., Luppe, S., \& Leite, M. I., et al. (2013). Distinction of seropositive NMO spectrum disorder and MS brain lesion distribution. Neurology, 80(14), 1330-7. [DOI:10.1212/WNL.0b013e3182887957] [PMID] [PMCID]

Comabella, M., \& Montalban, X. (2014). Body fluid biomarkers in multiple sclerosis. The Lancet Neurology, 13(1), 113-26. [DOI:10.1016/S1474-4422(13)70233-3]

Muramatsu, T. (2010). Midkine, a heparin-binding cytokine with multiple roles in development, repair and diseases. Proceedings of the Japan Academy, Series B, 86(4), 410-25. [DOI:10.2183/ pjab.86.410] [PMID] [PMCID]

Weckbach, L. T., Muramatsu, T., \& Walzog, B. (2011). Midkine in inflammation. The Scientific World Journal, 11 [DOI:10.1100/2011/517152] [PMID] [PMCID]

Salama, R. H., Muramatsu, H., Shimizu, E., Hashimoto, K., Ohgake, S., \&Watanabe, H., et al. (2005). Increased midkine levels in sera from patients with Alzheimer's disease. Progress in Neuro-Psychopharmacolooy and Biological Psychiatry, 29(4), 611-6. [DOI:10.1016/j.pnpbp.2005.01.018] [PMID]

Wu, G. C., Yuan, H., Pan, H. F., \& Ye, D. Q. (2017). Elevated plasma midkine and pleiotrophin levels in patients with systemic lupus erythematosus. Oncotarget, 8(25), 40181. [DOI:10.18632/oncotarget.13658] [PMID] [PMCID]

Maruyama, K., Muramatsu, H., Ishiguro, N., \& Muramatsu, T. (2004). Midkine, a heparin-binding growth factor, is fundamen- tally involved in the pathogenesis of rheumatoid arthritis. Arthritis \& Rheumatism, 50(5), 1420-9. [DOI:10.1002/art.20175] [PMID]

Shindo, E., Nanki, T., Kusunoki, N., Shikano, K., Kawazoe, M., \& Sato, H., et al. (2017). The growth factor midkine may play a pathophysiological role in rheumatoid arthritis. Modern Rheumatology, 27(1), 54-9. [DOI:10.1080/14397595.2016.11798 60] [PMID]

Kosugi, T., Yuzawa, Y., Sato, W., Arata-Kawai, H., Suzuki, N \& Kato, N., et al. (2007). Midkine is involved in tubulointerstitial inflammation associated with diabetic nephropathy. Laboratory Investigation, 87(9), 903-13. [DOI:10.1038/labinvest.3700599] [PMID]

Sato, W., Kadomatsu, K., Yuzawa, Y., Muramatsu, H., Hotta, N \& Matsuo, S., et al. (2001). Midkine is involved in neutrophil infiltration into the tubulointerstitium in ischemic renal injury. The Journal of Immunology, 167(6), 3463-9. [DOI:10.4049/ jimmunol.167.6.3463] [PMID]

Krzystek-Korpacka, M., Neubauer, K., \& Matusiewicz, M. (2010) Circulating midkine in Crohn's disease: Clinical implications. Inflammatory Bowel Diseases, 16(2), 208-15. [DOI:10.1002/ ibd.21011] [PMID]

Krzystek-Korpacka, M., Neubauer, K., \& Matusiewicz, M. (2009). Clinical relevance of circulating midkine in ulcerative colitis. Clinical Chemistry and Laboratory Medicine (CCLM), 47(9), 1085-90. [DOI:10.1515/CCLM.2009.248] [PMID]

Shaygannejad, V., Montazeri, S., Jamshidian, A., Tahani, S., Gharagozloo, M., \& Ashtari, F., et al. (2014). Correlation of midkine serum level with pro-and anti-inflamatory cytokines in multiple sclerosis. Iranian Journal of Immunology, 11(2), 1348. https://iji.sums.ac.ir/article_16774_00.html 